\title{
A domains-basedapproach to meeting social, emotional and mental health needs
}

\section{TIM O'BRIEN and AMELIA ROBERTS}

This article focuses on seven interacting research-informed domains. These domains are grounded in the research literature and have been applied in real world contexts to provide insight into, and an analytical tool for, meeting social, emotional and mental health (SEMH) needs within educational settings. Practitioner researchers applied the domains in a ten-month knowledge-exchange programme known as SWERL. Each domain is described in relation to the lived experience of teachers, pupils and schools. The article closes with a case study of how a primary school changed SEMH provision in the areas of 'planning transitions' and 'building relationships' and illuminates the benefits for children, staff and school culture.

\section{Introduction}

This context for this article is a ten-month knowledge-exchange and research programme, 'Supporting Wellbeing, Emotional Resilience and Learning' (SWERL), that is focused on supporting children and young people who experience social, emotional and mental health difficulties (SEMH). The Centre for Inclusive Education at UCL Institute of Education offers The SWERL programme to schools, a programme that is premised upon seven research-informed domains constructed by the authors. Schools use the domains as an analytic tool for insight, reflection, change and development. 
The term 'Knowledge Exchange' is often used to describe a process whereby researchers and practitioners work alongside each other in partnership, sharing their respective knowledge, ideas, evidence and expertise in order to achieve real world impact. During this process research questions and research priorities are co-constructed in order to support practitioner enquiry. Knowledge exchange also enables findings, often case studies, to feed back into the research cycle. Phipps and Morton (2013) challenge the linear nature of research when the underlying aim is the transfer of knowledge so that it will 'bridge the gap' between two communities. They see knowledge exchange, or the brokering of knowledge, as being essential in establishing a shared collaborative space in which to work.

This article includes a case study from the first iteration of SWERL. The second iteration of the programme is currently taking place in Suffolk. The authors and Dr Dennis Guiney, an associate of The Centre for Inclusive Education, work alongside schools supporting their SWERL research journey.

\section{Terminology}

We acknowledge that 'Social, Emotional and Mental Health Difficulties' [SEMH] (DfE, 2014) is an umbrella term that has been conceptualised in various ways by the UK Government over time. Recent descriptors include Emotional and Behavioural Difficulties (EBD) and Social, Emotional and Behavioural Difficulties (SEBD). Such umbrella terms in education have been criticised for their ontological fragility and for their potential to distract focus away from pedagogical processes and school systems onto within-child deficit (O'Brien, 2005). The current umbrella term, SEMH, has the intention of enabling children and young people to be identified within more precise definitions and understandings of difficulties and need, for example, relating to depression, trauma and anxiety. Although behaviour still remains a topic for discussion or concern in schools, it is no longer codified (O’Brien, 2018).

\section{A research-informed approach}

Our intent was to develop a research-informed domains-based approach to supporting children and young people who experience SEMH. To do so we referred to a scoping cross-cultural literature review focusing on identification, assessment and educational provision for children and young people who experience SEMH (Carroll and Hurry, 2018). The domains emerged from our analysis of 
this review and other literature in the field. The domains emanate from a biopsychosocial perspective. A biopsychosocial perspective, for example, considers that mental health difficulties and needs may originate from a biological cause, a psychological cause, an individual's social environment or from any combination of these. It provides the fullest perspective for understanding complexities - whilst also ensuring an analytic balance between considering within-child factors and within-environment factors. Adopting this perspective means that there are a number of different ways that a school can make meaning, search for solutions and meet additional needs.

\section{The seven domains}

We propose seven research-informed domains for supporting children and young people who experience SEMH and meeting their needs. We also highlight in this article how using a domains-based approach not only benefits those who experience SEMH or special educational needs and disabilities (SEND) but can also increase opportunities and participation for all children and young people. The seven domains are,
Supported and informed staff
Graduated Response to Need: role of the teacher
Enabling Environment
Whole School Coherence and Design
Building Relationships
Robust Communication Systems
Planning Transitions

Although the domains are presented here in list form, this does not indicate a hierarchy. Due to the nature of supporting pupils who experience SEMH and the complex ecology of schools, the domains are inevitably interlinked: there are many conceptual and real world overlaps between them. The domains enable schools to consider multi-faceted approaches to any particular issue, area of concern or focus for development. Whilst it may appear that any of the seven domains can self-exist, once schools use them as an analytic tool it rapidly becomes evident that they are relational. The seven domains are designed to interlock but they also have the capacity to unlock: a focus in one domain may unlock something that needs attention, change or development in another domain. 
We shall now consider each domain individually offering examples of how the domain is relevant to teachers, children, young people and families. Following this we provide a case study to illuminate how the domains can be applied to support pupils and staff and to develop school culture.

\section{Supported and informed staff}

We use the term 'staff' here as an inclusive term that involves teachers, teaching assistants and others. Simpson et al. (2011) emphasise that knowledgeable staff are a key component in effective provision for children and young people who experience SEMH. The literature also supports the view that staff must be informed and aware of how to support children and young people who present challenges. For example, relevant and meaningful professional development that has an impact on practice is essential. SEMH-specific and inclusion-focused continuing professional development for teachers and teaching assistants informs, develops and embeds responsive pedagogy and practice. In terms of support, 'teacher' wellbeing has to be taken seriously too. Teachers and teaching assistants may need specific support, such as coaching or supervision, where they are provided with the opportunity to reflect on, and manage, the emotional impact or stress that supporting those who experience SEMH can generate.

When reflecting on this domain, teachers have considered aspects such as how skilled staff are in de-escalating behaviour that is challenging, how aware staff are about the reasons why some children and young people present with behaviour that is challenging, the nature of continued professional development and its impact on culture and pedagogy, coaching as a form of support for staff, the impact of mental ill-health and access to relevant research for all staff.

\section{Graduated response to need - the role of the teacher}

In responding to need, interventions can be conceptualised as occurring at a universal level [with application to all students], at a secondary level [with application to some students] and at a tertiary level [with application to an individual student]. This corresponds with the 'graduated response to need' as described in the SEND Code of Practice (DfE, 2014). In terms of identification and assessment the literature highlights how initially this begins at a universal level for all children and young people who experience SEMH, with universal approaches common to both mainstream and specialist settings. Functional assessment enabling staff to understand the reasons why children and young people may 
behave the way they do - is a common approach at the universal stage. If universal provision is effective, fewer children will need targeted support and specialist interventions. Having analysed need from a universal level, schools may need to move through an increasingly more multi-method and personalised process, which can ultimately result in individualised specialist support that requires frequent review and adaptation. In the Code of Practice this is clearly expressed as a process of 'Assess, Plan, Do, Review' (DfE, 2014). For example, certain challenging behaviours or mental health conditions such as clinical depression may initially be understood through a universal level lens but specialist approaches, which could include therapeutic intervention, might be required for individuals. We may find that there will be a point in time in educational settings when analytic consideration of mental health needs may occur at the universal level foreveryone.

A core element of this domain involves focusing on inclusive culture and capacity: removing barriers to learning and increasing participation in learning. Inclusive pedagogy is one critical dimension of this domain. This domain also places a focus on clarity of guidance on how to assess whether or not children and young people require additional or individualised support, liaise with external providers, evaluate and select appropriate interventions and assess impact. The child and the family must be central to this process.

When reflecting on this domain, teachers have considered aspects such as what 'inclusive teaching' means in their own school context, person-centred planning, the role of teacher, pupil and parent/carer/family voice, the breadth of information and guidance available about intervention options, consideration of emotional factors that impact on learning and the nature of assessment practices.

\section{Enabling environment}

This domain does not only apply to the physical environment and how design features can make an environment enabling. An environment that is emotionally enabling is essential too, especially in the SEMH context. Here we begin by considering physical design. In 2008, the department for children, schools and families published 'Building Bulletin 102' (2008). This document offers comprehensive advice about the design of schools - and the design of spaces within schools - to support children and young people who experience SEND. The document also includes a range of case studies where inclusive design principles are explained in detail with reference to differing phases of education. In primary education the main emphasis is on how space can be used to promote learning, 
how the physical environment can promote a sense of wellbeing and how creating easy access to small group spaces and support spaces can enhance learning. The need for large open spaces that can be converted to a more 'cellular' arrangement when required is also highlighted. Updated guidance for specialist provision has been provided, which includes specific advice regarding SEMH.

Lippman (2015) considers how school design and architecture can enable learning, with particular reference to the Vygotskian zone of proximal development (ZPD). Vygotsky described the difference between a learner's actual level and their potential level as the ZPD. Lippman posits that pupils can progress through the ZPD and therefore learn more effectively when physical design offers space for collaboration but also provides the opportunity for working on your own when required.

Whilst physical design of the environment can enable access to learning, emotional design is also similarly important, especially so for those who experience SEMH. Relationship building is a fundamental aspect of emotional design within this domain. The empirical base validates that relationship building should be a whole domain in itself, therefore relationships will be discussed at a later point. Learning is not solely a cognitive process and therefore understanding social and emotional factors that can impact on learning is vital in supporting those who experience SEMH. For example, 'Attachment disorders' are referred to in the SEND Code of Practice (2014) as a specific element of SEMH that children and young people can experience that manifest in multiple ways and can create barriers to learning. As a response, some schools have become 'attachment aware' as a whole school initiative. A whole school audit and whole school training are components of the attachment aware initiative and the approach is rooted in an understanding of attachment theory (Bowlby, 1979). Some schools have also chosen to adopt mental health teaching programmes. Naylor et al. (2009) assessed the impact of a six-week mental health teaching programme in two secondary schools in the UK and found significant increases in empathy and sensitivity towards people who encounter and experience mental health difficulties as a result of the programme.

When reflecting on this domain, teachers have considered aspects such as flexible use of space in relation to expected and emerging need, appropriate space for restorative practice, movement times and flow around the school building, the physical, social and emotional nature of learning spaces, and involving children, young people, governors and parents in decisions about making environments more enabling. 


\section{Whole school coherence and design}

This domain focuses on systemic coherence and the way in which different elements of the whole school system partner and interplay. The coherence of a system is interlinked with the intended purpose of the system and in this context coherence would be evident in provision mapping and provision planning as well as in assessing whether the system is meeting the needs of children, families and staff members. Analysing systems through the lens that this domain provides will illuminate how aspects of the complex ecology of the system, such as supporting parents in order to build respectful partnerships, or working with transdisciplinary teams, are coherent or not in their aim of meeting additional needs. Auerbach (2012) proposes that promoting partnerships across the whole system is a "moral obligation" of school leadership.

One element of the whole school system, as understood within this domain, involves supporting parents. Here we assert that phrases such as "parents who are difficult to reach" deflect attention away from an analysis of the nature and role of schools and systems and therefore must be challenged. Cooper and Jacobs (2011) highlight how their international review of research studies relating to best practice in the field of SEMH illustrates that working supportively with parents is a robust preventative intervention. Inclusive and supportive approaches play a significant role in the development of a sense of trust and belonging for families in their relationships with schools (Francis et al., 2016).

Another aspect that is particularly evident in the literature is how important it is for systems to be designed so that they support working in a transdisciplinary manner. It is also important to reflect on the impact this has on how systems learn and on how systems understand what SEMH is and is not. Systems, even when they are designed to be inclusive, are not value-neutral and can still promote preferred ways of being (O'Brien, 2001). Reflecting on whole school coherence and design may require a degree of professional courage if teachers, especially early career teachers, are to challenge elements of a school system that may be incoherent and have an unintended negative effect on ethos, practice, initiatives or partnerships. Our experience of the SWERL programme highlights that teachers are willing to demonstrate professional courage as advocates for children and young people who are experiencing challenges.

When reflecting on this domain, teachers have considered aspects such as systemic memory, coherent entry and exit criteria for additional support, integrated 
policies, stakeholder engagement in resource allocation and how power dynamics impact on parental connection and involvement.

\section{Building relationships}

This domain places relationship building explicitly at the heart of school life. The importance of building and maintaining positive relationships between the teacher and pupil in promoting learning has almost folkloric status in education. However, there is a robust body of evidence to support this view and there is also evidence to support this view in relation to children and young people who experience SEMH and SEND.

The concept of building relationships includes establishing, evaluating, maintaining, nurturing and sometimes re-building relationships. Teacher and pupil relationships are a core element of this domain, but relationships within schools and their communities are multiple and dynamic. They include, staff-staff relationships, pupil peer-to-peer relationships and school-family relationships. There are also system-to-system relationships that need to be reviewed.

Cooper and Jacobs (2011) emphasise the importance and value of humanistic and non-coercive approaches. There is strong empirical support for the use of non-coercive approaches. Trusting and respectful relationships between staff and pupils have also been shown to reduce sanctions in a manner that is significant (Mowat, 2010). The stronger the relationship between staff and pupils, the more significant the decline in school sanctions. A meta-analysis of ninety-nine research studies highlights how person-centred teacher-pupil relationships can lead to improvements in engagement and achievement. This is substantially the case for pupils who are "from disadvantaged economic backgrounds and children with learning difficulties" (Roorda et al., 2011).

Other research studies support the proposition that the process of building relationships is fundamental to successful learning. Underpinning all successful interventions in the field of SEMH is a positive approach to children and young people who experience SEMH. A positive approach includes avoiding a deficit model - which includes looking no further than within-child factors or making judgmental assumptions about within-family factors - encouraging pupils to feel emotionally secure, demonstrating compassion and empathy and developing teacher-pupil relationships that are respectful. 
When reflecting on this domain, teachers have considered aspects such as identifying a key person who is the first point of contact for a vulnerable pupil, stress management for staff and pupils, embedding building relationships as a core element of school culture, investigating pupil voice and structured support relationships between older and younger pupils.

\section{Robust communication systems}

This domain specifically encourages schools to examine and analyse the purpose, effectiveness and outcomes of their systems, processes and interactions. Managing confidentiality, communication between school and home, safeguarding, data collection and clear role-responsibilities of core staff - for example, defining and clarifying the role of the SENCO (Fitzgerald and Radford, 2017) - exists within the systemic dimension of this domain.

Communication with parents, and how and when it occurs, is seen as an important aspect of supporting children and young people who experience SEMH. Ofsted (2009), through the analysis of a series of case studies, highlighted the characteristics of twelve "exceptionally successful" special schools and pupil referral units. Partnership with parents was seen as a key feature of all of these schools and this included effective communication. Robust communication with parents was seen to create strong school-home bonds. It was also acknowledged that developing partnerships with parents in such a context involves hard work.

In an evaluation of three parenting programmes for parents of children aged 8 to 13 in eighteen UK Local Authorities, Lindsay et al. (2011) indicate how pupil behaviour improved. Whilst some programmes were more effective than others, there was a significant reduction in "conduct problems" as an outcome of all parenting programmes. Communication with parents, which involves seeing the parental perspective, can have a positive impact on improving pupil behaviour. Extensive evidence exists that highlights the value of effective communication, connection and collaboration with parents. All schools contact parents but this does not mean that all schools connect with parents. Other dimensions involve planned and unplanned communication in relation to human interactions, which includes the type of language used by staff to support pupils who are distressed or anxious.

When reflecting on this domain, teachers have considered aspects such as clarity around roles and responsibilities, communication with key contact points in 
external agencies, the structure and nature of meetings with parents, readability and accessibility of communications with the wider school community and agreements about response times to parental communication.

\section{Planning transitions}

Macro and micro transitions of multiple types are always occurring within schools. What may appear to be ordinary or even mundane transitions can be problematic for some children and young people. Transitions might generate anxiety and stress; they can even create a sense of unpredictability when predictability is intended. Transitions can be problematic for children who have experienced trauma. Transitions can be challenging for looked-after children who may feel overwhelmed by constant transition in their lives. Change, both expected and unexpected, can increase feelings of anxiety, panic or even loss of control. This may drive behaviours that present as challenging.

Transitions can be physical and related to routine and non-routine movement and change within and around the environment but they can be psychological too. School-related psychological transitions can range from returning to classrooms and being ready to learn after lunch break, changing activities within a lesson or transition from a small rural primary school to a large secondary school. There are also life-stage transitions to consider that have a psychological impact on children and young people such as bereavement, moving house or the break-up of relationships. Therefore, the planning and understanding of transitions within schools and classrooms will always need attention and evaluation. For example, primary and secondary schools must and do look carefully at the transition process to secondary school, as there is agreement in the international literature that academic achievement can decline at this transitional point. Many factors may be relevant in this decline such as change in self-concept, diminishing self-confidence or a decreased interest in academic work during the onset of adolescence.

We have indicated that transitions can be problematic but we would also stress that teachers can plan transitions so that they enhance a child or young person's emotional growth and development, such as increasing their resilience. It is also important that planned transition processes are clearly communicated to, and understood by, teachers, pupils and families. Woolfolk and Margetts (2013) propose that while a particular focus tends to be placed on ensuring transitions work for particular children or groups of children, all children will benefit from a focus on good transition practice. 
When reflecting on this domain, teachers have considered aspects such as the use of visual timetables, transitions in teaching and learning, the transitional impact of life events, learning support transitions, placement breakdowns, transitions from one year group to another and transitions during different school terms - for example, supporting those children for whom Christmas is challenging due to poverty or family circumstance.

\section{A case study: planning transitions and building relationships}

This anonymised case study provides one illuminative example of how the domains can be used as a tool for analysis, development and change. The school in this case study is an age 3 to 11 , four-form entry, non-denominational, innercity, community primary school in a London borough. The school serves and represents a diverse community.

\section{What did the school do?}

The school SWERL team, comprised of four members of staff, began by leading a comprehensive whole school audit using the seven domains. This process highlighted that planning transitions and building relationships were the two interrelated domains that the research project would focus on.

The key transition that needed improved planning was the lunchtime session, mainly when children were returning from lunchtime into class. Behaviour report analysis showed that the majority of behaviour incidents were due to lunchtime arguments, with conflicts often remaining unresolved and spilling over into the afternoon timetable. This had a negative impact on behaviour and learning. Most of the pupils involved in these incidents were those identified as experiencing SEMH. A focus on the importance of pupil voice, all pupils, resulted in a survey that illuminated how a large number of pupils felt that lunchtime was the least positive time in the school day. School playground observations triangulated this data and supported pupil perceptions. Observations also highlighted how School Meals Supervisory Assistants (SMSAs) might require a different type of training from that they had previously undertaken, this time adopting a more empowering approach. It was felt that pupils needed further support during lunchtimes to learn how to get the best out of this time and to ensure that school values were evident outside the classroom as well as inside. Therefore, lunchtime was selected as the 
key space in the school day where planning transitions and building positive relationships were areas for further development.

An action plan was shared with all the staff and agreed. SMSA training needed to be different. All SMSAs were asked for their perspective regarding what they see and encounter in the playground, what it means to them and how they respond to what is happening. This was used to baseline their confidence and skills, explore their lived experience and understand how they felt they could make a more positive impact. How your own culture and belief systems influence the manner in which you understand and respond to behaviour that is challenging was also discussed. Communication styles were explored, as were approaches related to restorative practice. SMSAs were invited to understand and consider the life context and narratives of children who attend the school. They also explored how emotions can impact on a child's behaviour. They learned new playground games that they could play with the children or explain to the children and supervise.

In relation to the pupils contributing to how lunchtimes could improve, this was included into the school pastoral programme. The SEMH advisory teacher attended lessons to discuss with the children how they could help to improve their own lunchtime experience and build more positive relationships with SMSAs - as well as with other children and adults. Individual pupil support was offered where necessary.

\section{Outcomes}

Before their training the majority of SMSAs indicated in a survey that they were not confident in understanding and dealing with behaviour that was challenging. Lunchtime could be stressful for them, which affected their responses. After the SWERL project was completed, the majority indicated that they were now much more confident. They talked about no longer shouting at children, not judging children - especially based on a child's previous behaviour - and being better at both listening and responding. Their feedback identified that they were much calmer and, in some cases, they found that talking less could help to reduce stress in a challenging situation. They identified the value of having more knowledge and skills. Observations indicated that adults interacted more often and more positively with children.

SMSAs in this school are now given extra support, which includes time to meet and reflect on their practice. This was not the case before the SWERL project. 
There is a much clearer definition of the role and responsibilities of the SMSA and how they will be supported; there is now an identified member of staff available with whom they can discuss concerns and solutions. Children are also clearer about their responsibilities at lunchtime. Data analysis shows that playground incidents have reduced. During the project a governor engaged with the project and became the SWERL governor. This role continues as an aspect of school governance. Also, as a response to pupil voice, the school is planning to alter aspects of the outdoor play area to incorporate cost-effective themed play spaces.

The 'building relationships' domain also raised awareness of how the school could develop further provision for staff wellbeing. A staff wellbeing team was created, a survey was conducted and this resulted in wellbeing actions being taken. Space is now available for staff to meet to share their challenges and reflect on solutions.

\section{Conclusion}

The first iteration of the SWERL project offers early stage proof-of-concept support for the use of the seven domains in terms of application to, and meaning within, the real world context of schools. They have enabled schools to identify research projects and create meaningful actions, interventions and case studies. They have been shown to be beneficial in relation to meeting the needs of children and young people who experience SEMH and positive outcomes for all pupils have also been reported. The domains have promoted a way of looking that offers solutions and impact but, critically, they raise further questions too - as is their intention. Scrutiny of the robustness and the limitations of the domains, and the efficacy of their application in schools, continues as they are applied in new contexts.

\section{References}

AUERBACH, S. (2012) School Leadership for Authentic Family and Community partnerships: Research Perspectives for Transforming Practice. New York: Routledge.

BOWLBY, J. (1979) The Making and Breaking of Affectional Bonds. London: Routledge.

CARROLL, C. and HURRY, J. (2018) Supporting pupils in school with social, emotional and mental health needs: a scoping review of the literature. Emotional and Behavioural Difficulties, 23, 3, 310-325.

COOPER, P. and JACOBS B. (2011) Evidence of Best Practice Models and Outcomes in the Education of Children with Emotional Disturbances/Behavioural Difficulties. An International Review. NCSE, report, No7. 
DEPARTMENT FOR CHILDREN, SCHOOLS AND FAMILIES (2008) Designing for disabled children and children with special educational needs: Guidance for mainstream and special schools. BB102.

DFE (2014) Special educational needs and disability code of practice: 0 to 25 years. London: Crown.

FITZGERALD, J. and RADFORD, J. (2017) The SENCO Role in Post-Primary Schools in Ireland: Victims or Agents of Change? European Journal of Special Needs Education, 32, 3, 452-466.

FRANCIS, G.L., BLUE-BANNING, M., TURNBULL, A.P., HILL, C., HAINES, S.J. and GROSS, J.M. (2016) Culture in inclusive schools: Parental perspectives on trusting family-professional partnerships. Education and Training in Autism and Developmental Disabilities, 51, 3, 281-293.

LIPPMAN, P. (2015) Designing collaborative spaces for schools. Education Digest, 80, 5, 39-44.

LINDSAY, G., STRAND, S. and DAVIS, H. (2011) A comparison of the effectiveness of three parenting programmes in improving parenting skills, parental mental-wellbeing and children's behaviour when implemented on a large scale in community settings in 18 English local authorities: The parenting early intervention pathfinder (PEIP). BMC Public Health.

MOWAT, J.G. (2010) "He comes to talk to me about things": Supporting pupils experiencing social and emotional difficulties - a focus upon interpersonal relationships. Pastoral Care in Education, $28,3,163-180$.

NAYLOR, P., COWIE, H., WALTERS, S., TALAMELLI, L. and DAWKINS, J. (2009) Impact of a mental health programme on adolescents. British Journal of Psychiatry, 194, 4, 365-370.

OFSTED (2009) Twelve Outstanding Special Schools: excelling through inclusion. Crown.

O'BRIEN, T. (2001) Learning from the hard cases. In T. O'Brien (ed.) Enabling Inclusion: Blue skies...dark clouds? London: The Stationer Office.

O'BRIEN, T. (2005) Social, Emotional and Behavioural Difficulties. In A. Lewis andB. Norwich (eds), Special Teaching for Special Children? Pedagogies for Inclusion. Milton Keynes: Open University Press.

O'BRIEN, T. (2018) Reflections on 'Schools: An Evolutionary View'. Warnock Virtual Issue: NASEN https://onlinelibrary.wiley.com/page/journal/14679604/homepage/warnockvirtualissue\#/

PHIPPS, D. and MORTON, S. (2013) Qualities of knowledge brokers: reflections from practice. Evidence and Policy: A Journal of Research, Debate and Practice, 9, 2, 255-265.

ROORDA, D.J., KOOMEN, H., SPLIT, J. and OOORT, F.J. (2011) The influence of affective teacher-student relationships on student's school engagement and achievement: A meta-analytic approach. Review of Educational Research, 81, 4, 493-529.

SIMPSON, R.L., PETERSON, R.L. and SMITH, C.R. (2011) Critical Education Program Components for Students with Emotional and Behavioural Disorders: Science, Policy and Practice. Remedial and Special Education, 32, 3, 230-242.

WOOLFOLK, A. and MARGETTS, K. (2013) Educational Psychology (3rd Edition). Pearson: Australia.

\section{Correspondence}

Tim O'Brien

Psychology \& Human Development, UCL London20

Bedford Way

London WC1H 0AL

United Kingdom

Email: tim.o’brien@ucl.ac.uk 